\title{
BOUNDED HARMONIC BUT NO DIRICHLET-FINITE HARMONIC
}

\author{
BY YOUNG K. KWON \\ Communicated by F. W. Gehring, August 28, 1972
}

\begin{abstract}
The purpose of the present note is to announce that for each $n \geqq 3$ there exists a Riemannian $n$-manifold, which carries nonconstant bounded harmonic functions but no nonconstant Dirichletfinite harmonic functions.
\end{abstract}

1. A $C^{2}$-function $u$ on a Riemannian $n$-manifold $M$ is harmonic on $M$ if $\Delta u=0$, where

$$
\Delta u=\frac{-1}{g^{1 / 2}} \sum_{i, j=1}^{n} \frac{\partial}{\partial x^{i}}\left(g^{1 / 2} g^{i j} \frac{\partial u}{\partial x^{j}}\right) .
$$

Here $\left(g_{i j}\right)$ is the metric tensor for $M,\left(g^{i j}\right)=\left(g_{i j}\right)^{-1}$, and $g=\operatorname{det}\left(g_{i j}\right)$.

It is not known (cf. Sario-Nakai [4, p. 406]) whether or not for each $n \geqq 3$ there exists a Riemannian $n$-manifold $M$ which carries nonconstant bounded harmonic functions but every harmonic function $u$ on $M$ is a constant whenever its Dirichlet integral

$$
D(u)=\int_{M} \sum_{i, j=1}^{n} g^{i j} \frac{\partial u}{\partial x^{i}} \frac{\partial u}{\partial x^{j}} d V<\infty,
$$

where $d V=g^{1 / 2} d x^{1} \wedge d x^{2} \wedge \cdots \wedge d x^{n}$ is the volume element. For $n=2$ the problem was solved in the affirmative by Tôki [5], his example known as Tôki's example. Royden [2] and Sario [3] also obtained a similar result.

The purpose of the present note is to announce that for each $n \geqq 3$ there does exist a Riemannian $n$-manifold which solves the problem in the affirmative. ${ }^{1}$ Details will be published elsewhere.

2. Fix $n \geqq 3$. Denote by $M_{0}$ the punctured Euclidean $n$-space $R^{n}-0$ with the metric tensor

$$
g_{i j}(x)=|x|^{-4}\left(1+|x|^{n-2}\right)^{4 /(n-2)} \delta_{i j}, \quad 1 \leqq i, j \leqq n,
$$

where $|x|=\left[\sum_{i=1}^{n}\left(x^{i}\right)^{2}\right]^{1 / 2}$ for $x=\left(x^{1}, x^{2}, \ldots, x^{n}\right) \in M_{0}$.

Lemma. Every positive harmonic function $u$ on $M_{0}$ has the form:

AMS (MOS) subject classifications (1970). Primary 30A48.

Key words and phrases. Riemannian $n$-manifold, harmonic functions, Dirichlet integral, Tôki's surface.

${ }^{1}$ Professor Sario informed me that he recently obtained a similar result with Professors Wang and Hada. 


$$
u(x)=a /\left(1+|x|^{n-2}\right)+b
$$

for some constants $a, b$.

3. For each pair $(m, l)$ of positive integers $m, l$, and $k=z^{m-1}(2 l-1)-1$, set

$$
\begin{aligned}
& H_{m l}=\left\{8^{k} x=\left(8^{k} x^{1}, 8^{k} x^{2}, \ldots, 8^{k} x^{n}\right) \in M_{0}|| x \mid=1 \text { and } x^{1} \geqq 0\right\}, \\
& H_{m l}^{\prime}=\left\{8^{-k} x=\left(8^{-k} x^{1}, 8^{-k} x^{2}, \ldots, 8^{-k} x^{n}\right) \in M_{0}|| x \mid=1 \text { and } x^{1} \geqq 0\right\} .
\end{aligned}
$$

Denote by $M_{0}^{\prime}$ the manifold obtained from $M_{0}$ by deleting all the closed hemispheres $H_{m l}$ and $H_{m l}^{\prime}$.

Take two sequences $\left\{\boldsymbol{M}_{0}^{\prime}(l)\right\}_{1}^{\infty}$ and $\left\{\boldsymbol{M}_{0}^{\prime \prime}(l)\right\}_{1}^{\infty}$ of duplicates of $\boldsymbol{M}_{0}^{\prime}$. For each fixed $m \geqq 1$ and subsequently fixed $j \geqq 0$ and $1 \leqq i \leqq m$, connect $M_{0}^{\prime}(i+m j)$ with $M_{0}^{\prime \prime}(i+m+m j)$ for even $j$ and $M_{0}^{\prime}(i+m j)$ with $M_{0}^{\prime \prime}(i-m+m j)$ for odd $j$, crosswise along all the hemispheres $H_{m l}$ and $H_{m l}^{\prime}(l \geqq 1)$. The resulting Riemannian $n$-manifold $N$ is an infinitely sheeted covering manifold of $M_{0}$.

THEOREM. For the manifold $N$ the following are true:

$$
\text { (1) } \operatorname{dim} H B(N)=2, \quad \text { (2) } \operatorname{dim} H D(N)=1,
$$

where $H$ is the space of harmonic functions, $D$ the space of Dirichlet-finite functions, $B$ the space of bounded functions and $H X$ stands for $H \cap X$.

It can be shown that every bounded harmonic function $u$ on $N$ takes the same value at all the points in $N$ which lie over the same point in $M_{0}$. Therefore the function $u$ is Dirichlet-finite only if $u$ is a constant. Here we use the $q$-Lemma (cf. Rodin-Sario [1, p. 39]).

\section{REFERENCES}

1. B. Rodin and L. Sario, Principal functions, Van Nostrand, Princeton, N.J., 1968. MR 37 \# 5378.

2. H. L. Royden, Some counterexamples in the classification of open Riemann surfaces, Proc. Amer. Math. Soc. 4 (1953), 363-370.

3. L. Sario, Positive harmonic functions, Lectures on functions of a complex variable, Univ. Mich. Press, 1955, 257-263.

4. L. Sario and M. Nakai, Classification theory of Riemann surfaces, Die Grundlehren der math. Wissenschaften, Band 164, Springer-Verlag, Berlin and New York, 1970. MR 41 \# 8660 .

5. Y. Tôki, On the examples in the classification of open Riemann surfaces. I, Osaka Math. J. 5 (1953), 267-280. MR 15, 519.

Department of Mathematics, University of TeXas, Austin, Texas 78712 